"DNA-controlled assembly of protein-modified gold nanocrystals." Cobbe, S.; Connolly, S.; Nagle, L.; Eritja, R.; Fitzmaurice, D.; Ryan, D. J. Phys. Chem. B, 107(2), 470-477 (2003). doi: 10.1021/jp021503p

\title{
DNA-Controlled Assembly of Protein-Modified Gold Nanocrystals
}

\section{Stephen Cobbe, Stephen Connolly, Declan Ryan, Lorraine Nagle, Ramon Eritja, ${ }^{\dagger}$ and Donald Fitzmaurice*}

Department of Chemistry, National Univerrsity of Ireland, Dublin, Belfield, Dublin 4, Ireland, and ${ }^{\dagger}$ Centro de Investigación y Desarrollo (CSIC), Jorge Girona 18-26, 08034 Barcelona, Spain

* Author to whom correspondence should be addressed

\begin{abstract}
.
The controlled assembly in solution of gold nanocrystals modified by attachment of complementary protein- DNA conjugates is described. The size of the aggregates formed can be controlled by the addition of singlestranded DNA, which quickly terminates the assembly process. The rate of formation of the aggregates can also be controlled by varying the salt concentration. Consequently, two distinct regimes of aggregation kinetics are observed. At low salt concentrations, aggregation is shown to be dependent on the rate of duplex formation between the modified gold nanocrystals, i.e., reaction-limited. At higher salt concentrations, aggregation is shown to be dependent only on the rate of diffusion of the nanocrystals, i.e., diffusion-limited. The results presented provide important insights into the rates of formation of nanocrystal assemblies. Moreover, the approach adopted is modular, requiring only the relevant biotin linker chemistry to be developed for a given nanoparticle, while also precluding unfavorable interactions between the DNA and the streptavidin-coated nanoparticle. The ability to control the rate of formation and size of nanocrystal aggregates assembled is important new knowledge. Application of this knowledge will inform future studies of nanocrystal assembly in solution involving different types of nanocrystals, which is of increasing technological significance.
\end{abstract}

\section{Introduction}

Recent advances in the synthesis and characterization of size monodisperse nanocrystals have led to insights into the unique optical and electronic properties of these materials $(1,2)$. It has been suggested that the ability to control the assembly of nanocrystals in solution and at patterned substrates will allow aspects of the technological potential of these materials to be fully exploited. A number of key developments, which represent progress toward these goals, have been described (3-22). One such 
development involves using high-information-content biomolecules, including DNA, to program the assembly of nanocrystals in solution.

DNA molecules have previously been used as linker and template molecules for the assembly of nanocrystals (23, 24). For example, gold nanocrystals have been surface-modified by oligonucleotides that are capable of recognizing and selectively binding part of the linker or template oligonucleotide strand. This approach has led to novel arrangements of nanocrystals and has also provided a basis for binary nanocrystal assembly in solution (25-28). Related work based on protein-DNA conjugates, similar to those used in this paper, has also enabled chemists and molecular biologists to assemble and uniquely define the position of nanocrystals within nanocrystal-protein- DNA assemblies (29-31).

The inherent recognition properties of biological macromolecules, such as DNA and proteins, and the associated diversity of interactions and binding energies, ranging from hydrogen bonding to covalent bonding, should allow the self-assembly of complex nanoscale structures whose complexity approaches those found in nature. In order, however, to successfully use biological macromolecules to program the assembly of these complex nanoscale structures in solution, a significant degree of control over the size and order of the nanocrystal assemblies will be necessary. If this can be achieved, the promise of the outlined approach may become possible.

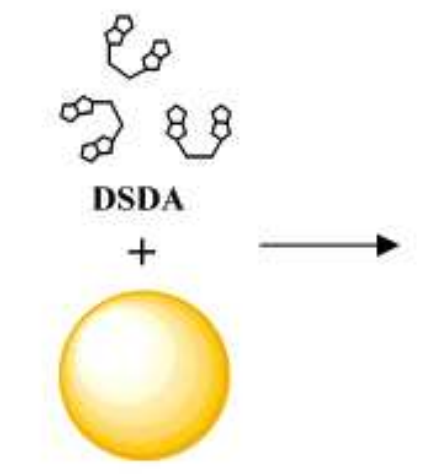

Gold Nanocrystals

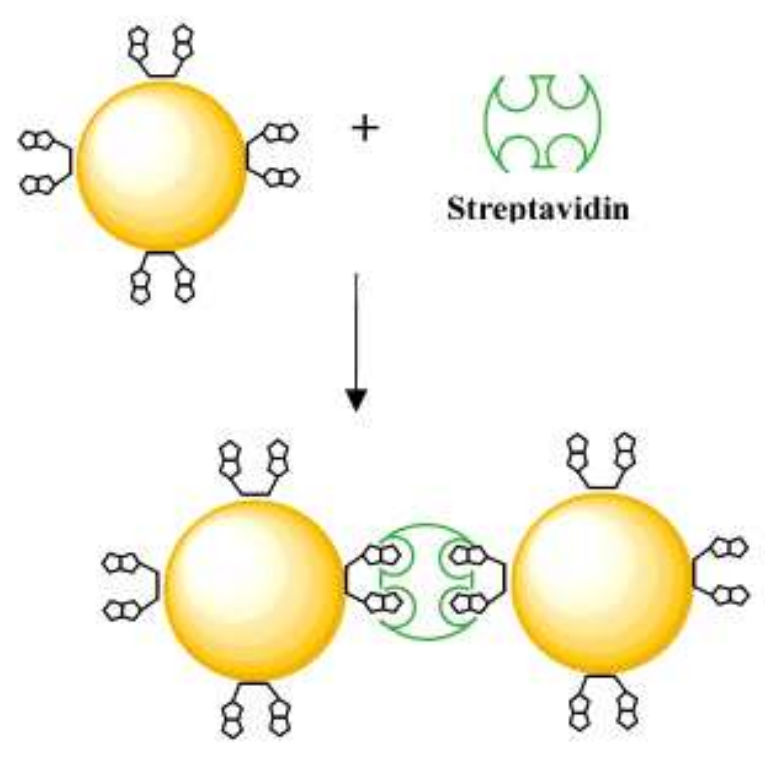

\section{SCHEME 1: Gold Nanocrystals Are Modified by Chemisorption of DSDA. Subsequent Addition of Streptavidin Leads to Aggregation of the Dispersion}

In this context, we recently developed a system based on the streptavidin-mediated assembly of biotin-modified gold nanocrystals, which has previously been reported (Scheme 1) (32). A model of this aggregation 
has also been developed which is an extension of both the classical Smoluchowski model of aggregation and a more recently developed model of linker induced aggregation by Kisak et al. (33-35).

In this paper, we report a unique approach to the self-assembly of gold nanocrystals in solution using DNA oligomers (Scheme 2). By modifying gold nanocrystals with a biotin analogue, the nanocrystals are programmed to recognize and bind selectively streptavidin-DNA conjugates. The addition of gold nanocrystals, similarly programmed to recognize and bind selectively the complementary streptavidin-DNA conjugate, is expected to result in nanocrystal assembly. DNA duplex formation between the two complementary DNA oligomers bound to the individual nanocrystals drives the assembly process. The addition of a DNA linker or template molecule to initiate the assembly process is also unnecessary.

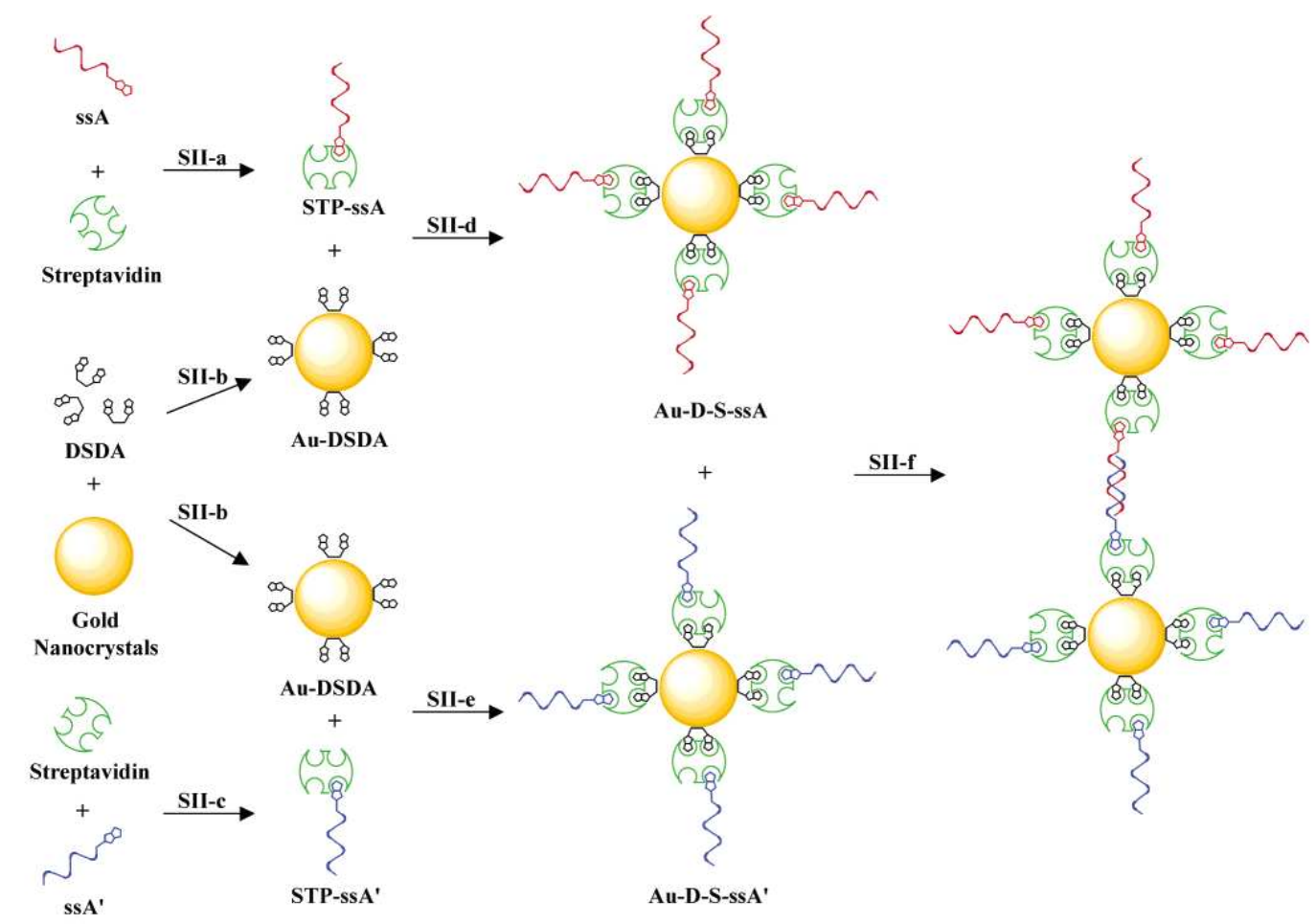

SCHEME 2: Gold Nanocrystals Are Modified by Chemisorption of DSDA. They Are Further Modified by Addition of Complementary Streptavidin-DNA Conjugates to Separate Sets of Nanocrystals. Subsequent Combination of Both Sets of Nanocrystals Results in Duplex Formation between the Complementary DNA-Modified Nanocrystals and Aggregation of the Dispersion

This unique approach allows the kinetics of nanocrystal assembly to be controlled. Moreover, it is possible to terminate aggregation of a dispersion of nanocrystals at any time by the addition of single-stranded DNA (ssDNA) oligomers. Consequently, it is possible to control the rate of formation and size of the resultant assemblies.

Finally, it should be noted that the approach adopted is modular, requiring only the relevant biotin linker chemistry to be developed for a 
given nanoparticle, while also precluding unfavorable interactions between the DNA and the streptavidincoated nanoparticle.

\section{Experimental Section}

All chemicals were used as supplied by the Sigma-Aldrich Chemical Co. Ltd, unless otherwise stated. The disulfide desthiobiotin analogue (DSDA) was prepared as previously described.33 Distilled deionized Millipore-MilliQ water $\left(18 \Omega \mathrm{cm}^{-1}\right.$ ) filtered through a $0.02 \mu \mathrm{m}$ inorganic Anotop filter was used to prepare all solutions.

Preparation of Oligonucleotides. The 5'-biotin-hexaethylene glycol-oligonucleotides were prepared using an automatic DNA Synthesizer (ABI Model 392) and a LV200 synthesis cycle (Applied Biosystems, USA). Biotin-hexaethylene glycol moieties were introduced at the 5'-terminus of the 20 base-pair synthetic oligonucleotides by using hexaethylene glycol phosphoramidite (Glen Research, USA) and biotin phosphoramidite (Applied Biosystems, USA). The polystyrene supports used were LV200 columns (Applied Biosystems, USA). The coupling time of the biotin and the hexaethylene glycol phosphoramidites was increased from 30 to $480 \mathrm{~s}$. After the assembly of the sequences, the oligonucleotide supports were treated with $32 \%$ aqueous ammonia at $55{ }^{\circ} \mathrm{C}$ for $16 \mathrm{~h}$. The ammonia solutions were concentrated to dryness and the products were purified by reverse-phase HPLC. Oligonucleotides were synthesized with the last DMT group at the $5^{\prime}$-end to help reverse-phase purification. The sequences prepared were: 5'-biotin-hexaethylene glycol-CAG TAG TCA GTA GTA GTC AG-3' (ssA); 5'-biotin-hexaethylene glycol-CTG ACT ACT ACT GAC TAC TG $3^{\prime}$ (SSA'). Following the oligonucleotide synthesis, both sets of oligonucleotides were lyophilized and stored at $-20{ }^{\circ} \mathrm{C}$ prior to use.

UV-Visible Spectroscopy. UV-visible spectroscopy was performed using a HP-8452A Spectrophotometer using LabView software written to acquire data. All UV-visible spectra were referenced using appropriate solution backgrounds.

Dynamic Light Scattering. Dynamic light scattering (DLS) experiments were performed using a Malvern PCS-4700 instrument equipped with a 256-channel correlator. The laser source was the $488.0 \mathrm{~nm}$ line of a Coherent Innova-70 Ar ion laser typically at a power of $50 \mathrm{~mW}$. The temperature was maintained at $20{ }^{\circ} \mathrm{C} \pm 0.02{ }^{\circ} \mathrm{C}$ by an external circulator. Data treatment was as previously described (36). Scattered light was monitored at an angle of $90^{\circ}$.

Transmission Electron Microscopy. Transmission electron microscopy (TEM) was performed on a JEOL JEL-2000 EX electron microscope at an accelerating voltage of $80 \mathrm{kV}$. Samples were prepared by evaporating a drop of the aqueous dispersion on a carbon-coated copper grid.

Preparation of Buffers. Buffer A consists of $8.75 \mathrm{~mL}$ of a $1 \% \mathrm{w} / \mathrm{v}$ aqueous citrate solution diluted to $250 \mathrm{~mL}, \mathrm{pH} 7.2$. Buffer B consists of aqueous $10 \mathrm{mM}$ phosphate, $0.1 \% \mathrm{w} / \mathrm{v}$ Tween20, $2 \mathrm{mM}$ sodium azide 
solution, $\mathrm{pH}$ 7.2. Buffer $\mathrm{C}$ consists of a $10 \mathrm{mM}$ phosphate, $1 \mathrm{M} \mathrm{NaCl}, 0.1 \%$ $\mathrm{w} / \mathrm{v}$ Tween20, $2 \mathrm{mM}$ sodium azide solution, $\mathrm{pH}$ 7.2.

Preparation of Disulfide Desthiobiotin Analogue-Modified Gold Nanocrystals (Au-DSDA). Gold nanocrystal dispersions with narrow size distributions (approximately $16 \mathrm{~nm}$ ) were prepared by a modification of the Frens method as previously described (32). Modification of the gold nanocrystals with DSDA was carried out by the addition of an aliquot of DSDA solution in Buffer A (14 $\mu \mathrm{M}, 5 \mathrm{~mL})$ to the gold sol ( $2 \mathrm{nM}, 250 \mathrm{~mL})$. The dispersion was sonicated (Ultrawave, USA) for $1 \mathrm{~h}$ and shaken on a minishaker-vortex (IKA Works Inc., USA) for $1 \mathrm{~h}$. The modified gold sol, referred to as Au-DSDA, was concentrated by centrifugation (9000 rpm, 25 min; Sorvall, SS34-rotor), briefly sonicated ( $<5 \mathrm{~s})$, and then filtered using a $0.1 \mu \mathrm{m}$ Anotop syringe filter membrane. The concentrated sample was redispersed in Buffer $A$ and characterized by UV-visible spectroscopy (for the Au-DSDA sol and the resulting modified nanocrystal dispersions an extinction coefficient of $4.712 \times 10^{8} \mathrm{~L} \mathrm{~mol}^{-1} \mathrm{~cm}^{-1}$ at $518 \mathrm{~nm}$ was used to determine the nanocrystal concentration), DLS and TEM.

Preparation of Streptavidin Solutions. Streptavidin was dissolved in deionized Milli-Q Millipore water to a final concentration of $80 \mu \mathrm{M}$ and stored at $4{ }^{\circ} \mathrm{C}$. The activity of the resulting solution was subsequently determined by titration using UV-visible spectroscopy (36). All other streptavidin solutions were prepared using Buffer $\mathrm{A}$ and stored at $4{ }^{\circ} \mathrm{C}$ prior to use.

Preparation of Oligonucleotide Solutions (ssA, SsA'). The lyophilized oligonucleotides were dissolved in Buffer $A$, unless otherwise stated, and stored at $4{ }^{\circ} \mathrm{C}$ prior to use. The concentrations of the resulting solutions were based on an extinction coefficient of $2.080 \times 10^{5} \mathrm{~L} \mathrm{~mol}^{-1} \mathrm{~cm}^{-1}$ for SSA and $1.856 \times 10^{5} \mathrm{~L} \mathrm{~mol}^{-1} \mathrm{~cm}^{-1}$ for SSA' at $260 \mathrm{~nm}$ (38).

Preparation of Streptavidin-Oligonucleotide Conjugates (STPSsA, STP-ssA'). The 1:1 streptavidin-oligonucleotide conjugate, STP-ssA, was prepared by the addition of a solution of SsA $(2 \mu \mathrm{M}, 500 \mu \mathrm{L})$ to a solution of streptavidin $(2 \mu \mathrm{M}, 500 \mu \mathrm{L})$ in a $3 \mathrm{~mL}$ glass vial. The resulting solution was agitated using the minishaker for $24 \mathrm{~h}$ at room temperature. During this period, the solution was sonicated briefly $(<5 \mathrm{~s})$ at hourly intervals to prevent nonspecific adsorption of the streptavidin to the glass. The 1:1 streptavidin-oligonucleotide conjugate, STP-ssA', was prepared in a similar manner.

Preparation of Au-DSDA-Streptavidin-Oligonucleotide Adducts (Au-D-S-ssA, Au-D-S-ssA'). To a solution of concentrated Au-DSDA (20 $\mathrm{nM}, 1 \mathrm{~mL})$ in a glass vial was added STP-ssA $(1 \mu \mathrm{M}, 1 \mathrm{~mL})$. The dispersion was agitated for $24 \mathrm{~h}$ at room temperature, sonicated briefly, and then centrifuged (9000 rpm, $25 \mathrm{~min}$ ). Following removal of the supernatant, the dark red precipitate was briefly sonicated and washed once with Buffer $A$ using centrifugation (9000 rpm, $25 \mathrm{~min})$. Streptavidin $(2 \mu \mathrm{M}, 500 \mu \mathrm{L})$ was added, and the vial was agitated for $12 \mathrm{~h}$. The dispersion was sonicated, centrifuged, and washed (x2) using Buffer A, as described above. An aliquot of ssA $(2 \mu \mathrm{M}, 1 \mathrm{~mL})$ was then added, and the resulting dispersion was 
agitated for $24 \mathrm{~h}$ and washed (x4) with Buffer $\mathrm{A}$ as before. The dispersion was finally filtered through a $0.1 \mu \mathrm{M}$ Anotop syringe filter and made up to 1 $\mathrm{mL}$ with Buffer $\mathrm{A}$. This dispersion will be referred to as Au-D-S-ssA. The corresponding Au-DSDA-streptavidin- oligonucleotide adduct, Au-D-S-ssA', was prepared similarly. Both adducts were characterized using UVvisible spectroscopy, DLS, and TEM. The concentration of each dispersion was approximately $20 \mathrm{nM}$.

DNA-Programmed Assembly of Protein-Modified Gold Nanocrystals. DLS was used to characterize the DNAprogrammed assembly of the protein-modified gold nanocrystals by monitoring the increase in the hydrodynamic radius following the addition of Au-D-S-ssA to Au-D-S-ssA'. Typically, an aliquot of Au-D-S-ssA' (20 nM, $25 \mu \mathrm{L}$ ) was added to a solution containing Au-D-S-ssA $(20 \mathrm{nM}, 25 \mu \mathrm{L}), 850 \mu$ í of Buffer B, and $100 \mu \mathrm{L}$ of Buffer C, giving a final salt concentration of 100 $\mathrm{mM}$. Samples for TEM were prepared immediately after aggregation by removing the relevant sample from the DLS cell.

To establish that DNA duplex formation is the principle driving force for the assembly process, a series of control experiments were also performed. First, an aliquot of Au-DS- ssA $(20 \mathrm{nM}, 25 \mu \mathrm{L})$ was added to a dispersion containing Au-D-S (20 nM, $25 \mu \mathrm{L})$, which corresponds to AuDSDA coated with a monolayer of streptavidin (33), in $850 \mu \mathrm{L}$ of Buffer B and $100 \mu \mathrm{L}$ of Buffer C. A similar control experiment, at corresponding concentrations and volumes, was performed by the addition of Au-D-S-ssA' to a dispersion of Au-D-S in $85 \mu \mathrm{L}$ of Buffer B and $100 \mu \mathrm{L}$ of Buffer C. For this experiment, it was anticipated that omission of the complementary oligomer on either set of modified nanocrystals would result in no assembly. This is a consequence of the complementary oligomer not being present, hence no duplex formation is possible.

Further control experiments involved the addition of an aliquot of AuD-S-ssA' $(20 \mathrm{nM}, 25 \mu \mathrm{L})$, after $3000 \mathrm{~s}$ of aggregation, to a dispersion containing Au-D-S-ssA $(20 \mathrm{nM}, 25 \mu \mathrm{L})$ and $\mathbf{s s A}^{\prime}(2 \mu \mathrm{M}, 25 \mu \mathrm{L})$ in $825 \mu \mathrm{L}$ of Buffer $B$ and $100 \mu \mathrm{L}$ of Buffer $C$. It would be expected that no aggregation would be observed since the preformed duplexes between Au-D-SssA and SsA' would ensure that Au-D-S-ss $\mathbf{A}^{\prime}$ is unable to bind to and form duplexes with Au-D-S-ssA. A similar experiment, at corresponding concentrations, volumes, and times, was performed by the addition of Au-D-S-ssA to a dispersion of Au-D-S-ss' $\mathbf{A}^{\prime}$ and SsA.

Time-Dependence of Size Distribution on Aggregation Termination. To terminate the aggregation process and investigate the resulting size distributions of the aggregates formed, ssDNA was added to an aggregating solution of complementary nanocrystals at various stages of the aggregation process. The addition of ssDNA was carried out after 600, 1500 , and $2700 \mathrm{~s}$ of aggregation. Typically, this was achieved by the addition of a solution of either SSA or SSA' $(2 \mu \mathrm{M}, 25 \mu \mathrm{L})$, in Buffer $\mathrm{B}$, to a dispersion containing both Au-D-S-ssA (20 nM, $\left.25 \mu{ }^{\prime} L\right)$ and Au-D-S-ssA' (20 nM, $25 \mu$ íL) in $850 \mu$ íL of Buffer B and $100 \mu \mathrm{L}$ of Buffer C. DLS was used to monitor the aggregation termination processes, and representative TEM samples were taken from the DLS cell upon completion of the experiments. 
Salt and Nanocrystal Concentration Dependence on Aggregation. To measure the effects of the salt and nanocrystal concentrations on the rate of assembly both parameters were varied independently of one another. DLS was used to characterize the respective aggregation processes as both parameter were varied. First, the aggregation of the complementary nanocrystals was monitored at 15, 25, $50,100,200,400$, and $600 \mathrm{mM}$ salt by adjusting the volume ratio of Buffer $B$ to Buffer $C$. For this experiment, the nanocrystal concentration was the same as for the preceding section. Second, experiments where the nanocrystal concentration was varied were carried out at $100 \mathrm{mM}$ salt. Aggregation was monitored at half and twice the nanocrystal concentrations used in the preceding section.

(a)

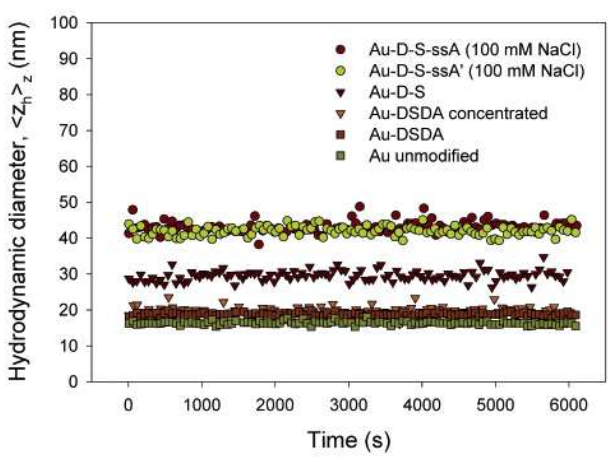

(c)

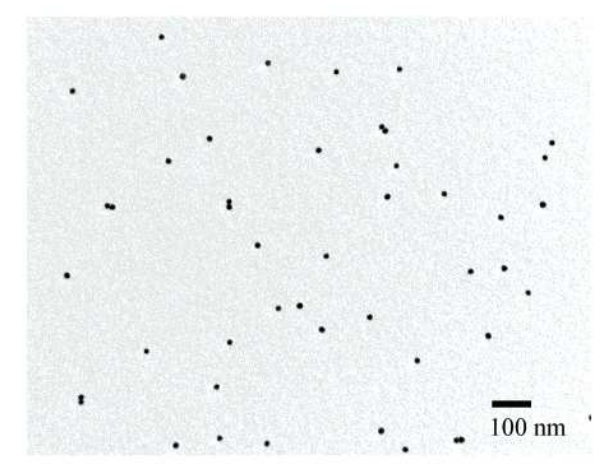

(b)

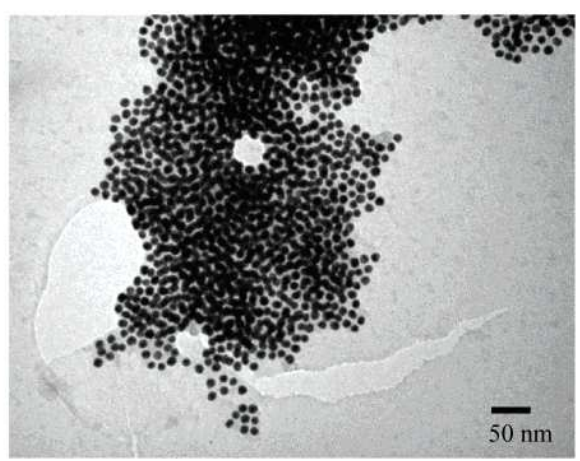

(d)

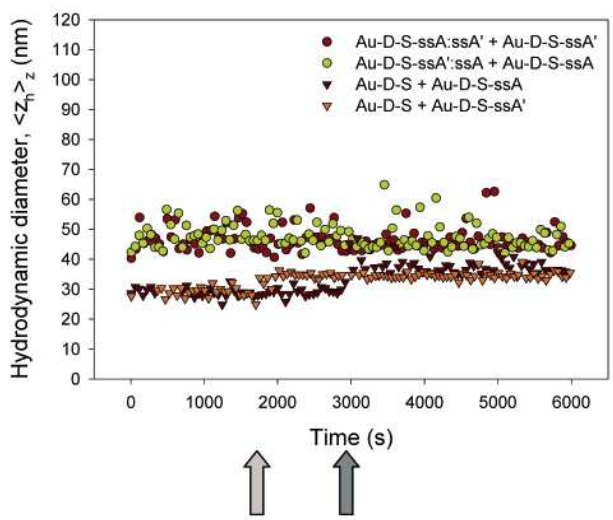

Figure 1. (a) Average hydrodynamic diameter as a function of time following successive modifications of the gold sol. (Note: The average hydrodynamic diameter values obtained for Au-D-S-ssA and Au-D-S-ssA' in Buffer A correspond to the values shown for the same adducts at $100 \mathrm{mM}$ salt concentration). (b) TEM image of a dispersion of Au-D-S-ssA and Au D-S-ssA' aggregated at $100 \mathrm{mM}$ salt concentration. (c) TEM image of AuDSDA further modified with streptavidin-DNA conjugate STP-sSA. A similar image was obtained for the modification of Au-DSDA with STP-ssA'. (d) Average hydrodynamic diameter as a function of time following addition of Au-D-S-ssA' after 3000 s to a dispersion containing Au-D-S-ssA and SsA'. Au-D-S-ssA was also added after 3000 s to a dispersion containing Au-DS-ssA' and SsA (See Supporting Information for a graphical illustration of 
this experiment). Also shown is the average hydrodynamic diameter as a function of time following addition of Au-DS- SsA to Au-D-S, and the addition of $\mathbf{A u}-\mathbf{D}-\mathbf{S}-\mathbf{s s} \mathbf{A}^{\prime}$ to $\mathbf{A u}-\mathbf{D}-\mathbf{S}$. The arrows indicate the time of addition of Au-D-S-ssA (dark gray) and Au-DS- ssA'(light gray). All solutions were at a salt concentration of $100 \mathrm{mM}$.

\section{Results and Discussion}

The following paragraphs detail the characterization of gold nanocrystals modified by protein-DNA conjugates. The assembly of the nanocrystals, mediated by DNA duplex formation, and subsequent insights into the assembly process are outlined. Specifically, factors that control the rate of growth and the size of the resultant aggregates are identified.

Streptavidin-Oligonucleotide-Modified Gold Nanocrystals. The assembly of DSDA-modified gold nanocrystals using the tetrameric protein streptavidin as a linker has previously been characterized (Scheme 1). It has been calculated that there are approximately 150 DSDA molecules per nanocrystal, and that 50 streptavidins per nanocrystal correspond to monolayer coverage (33). An initial increase of $2 \mathrm{~nm}$ in the size of the nanocrystals from 16 to $18 \mathrm{~nm}$ is observed upon the addition of DSDA (Figure 1a). This increase corresponds to a perpendicular alignment of the DSDA molecule (approximately $1.5 \mathrm{~nm}$ in length) on the surface of the charge-stabilized gold nanocrystals (Scheme 1 ).

In this work, the DSDA-modified gold nanocrystals are subsequently modified with a streptavidin-oligonucleotide conjugate (Scheme 2; SII-a, SII-c) and a further increase in diameter from 18 to $42 \mathrm{~nm}$ is observed (Figure 1a). This $24 \mathrm{~nm}$ increase in the overall diameter corresponds to the binding of an entity approximately $12 \mathrm{~nm}$ in length to the surface of the DSDA-modified gold (Scheme 2; SII-d, SII-e). This finding is consistent with the facts that the dimensions of streptavidin are $4 \times 4.2 \times 5 \mathrm{~nm}$ (39), while those of the biotin-hexaethylene glycol-oligonucleotides, ssA and ssA', are approximately $8.5 \times 2 \mathrm{~nm}$ (40).

Streptavidin itself is a tetrameric protein with four binding sites, and the arrangement of these sites is such that two adjacent pairs exist on opposite faces of the protein. Consequently, for the $1: 1$ conjugates, STPSSA and STP-sSA', the biotinhexaethylene glycol-oligonucleotides are free to bind to either site available on either face of the protein. Two adjacent sites will remain unoccupied on the unbound face, which can bind to the DSDA-modified gold because they are sterically unhindered (Scheme 2; SII-d, SII-e). The length of such a streptavidin- DNA conjugate is approximately $12.5 \mathrm{~nm}$ along the major axis. Hence, the binding of an entity representative of such dimensions corresponds to the observed increase in size. 
(a)

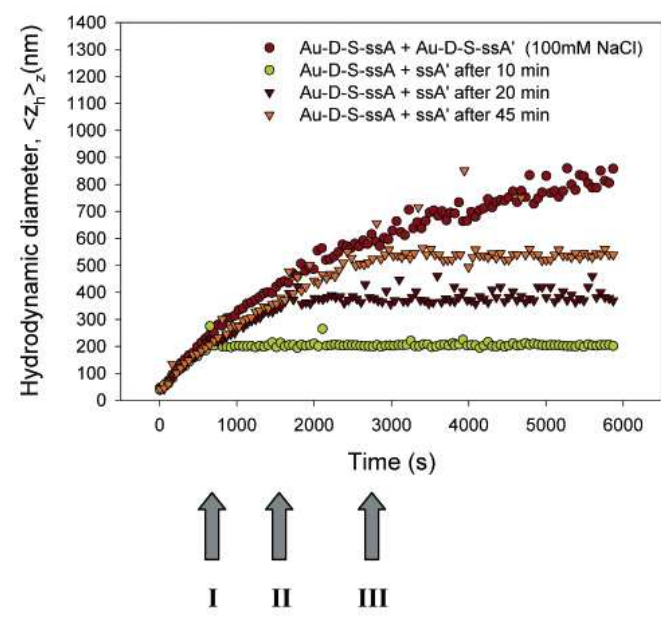

(b)-II

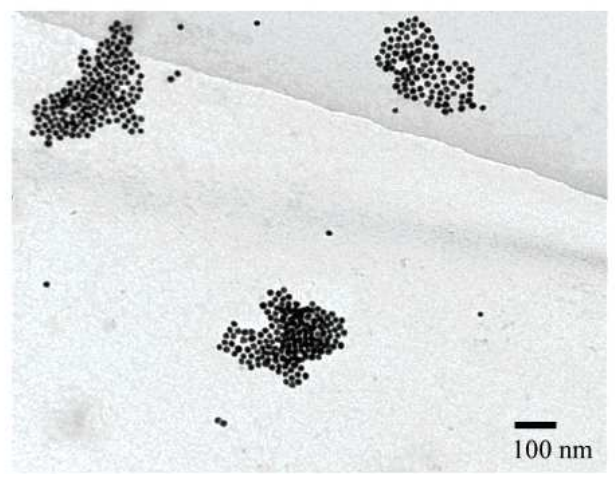

(b)-I

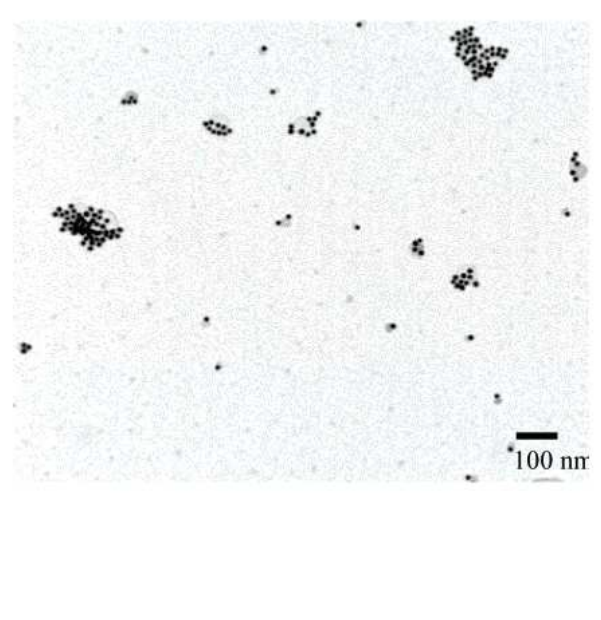

(b)-III

Figure 2. (a) Average hydrodynamic diameter as a function of time following addition of SsDNA oligomer to an aggregating dispersion of Au- DS-ssA and Au-D-S-ssA'. The arrows indicate the time of addition of ssDNA oligomer after (I) 600, (II) 1500, and (III) 2700 s of aggregation. (Note: Similar results were obtained for the addition of either SSA or SSA' oligomers). (b) TEM images corresponding to the addition of SSDNA oligomer after (I) 600, (II) 1500, and (III) $2700 \mathrm{~s}$ of aggregation.

Programmed Assembly of Protein-DNA Modified Gold Nanocrystals. The addition of Au-D-S-ssA to an equimolar dispersion of Au-D-S-ssA', both characterized as described above, at $100 \mathrm{mM}$ salt concentration yields a wine-colored precipitate within $1 \mathrm{~h}$ (Figure 1b). To establish that DNA is the driving force for the aggregation, a series of control experiments were performed. Addition of Buffer $\mathrm{C}$ to separate samples of Au-D-S-ssA and Au-D-S-ssA', up to a salt concentration of 100 $\mathrm{mM}$, resulted in no increase in the size of the respective samples (Figure 1a). Representative TEM images from the same samples (Figure 1c) show at most dimer and trimer formation.

Previous studies would suggest that such entities are formed by trace-free streptavidin, at which ssDNA is not bound, crosslinking DSDAmodified gold. This trace-free streptavidin is introduced when the $1: 1$ conjugates, STP-sSA and STP-sSA', are added to the DSDA-modified gold.33 In this instance, although the salt concentration is sufficient for DNA 
duplex formation and hence particle aggregation, no increase in size was observed. From this, one can conclude that the DNA strands are non selfcomplementary.

(a)

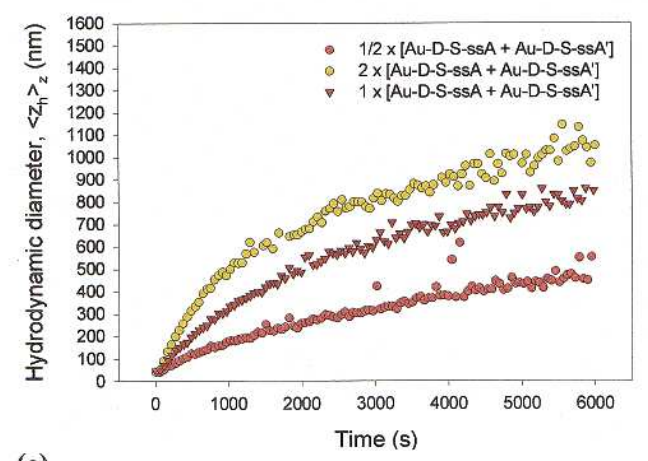

(6)

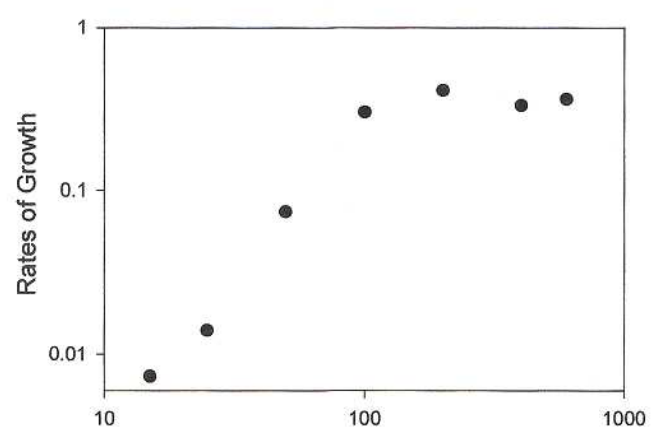

(b)

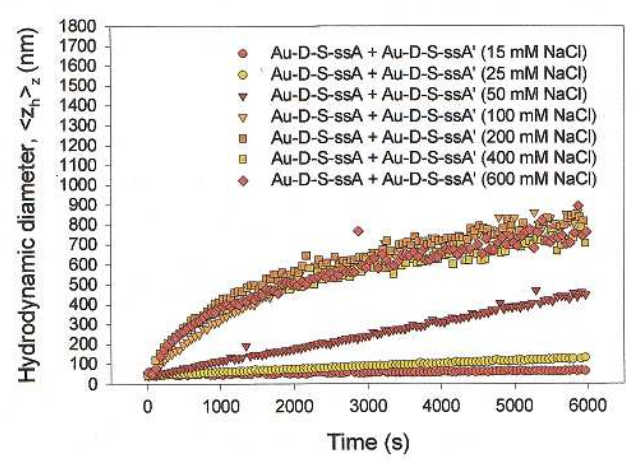

Figure 3. (a) Average hydrodynamic diameter as a function of time for an aggregating dispersion of Au-D-S-ssA and Au-D-S-ssA' at various nanocrystal concentrations. (Note: Nanocrystal concentrations are given relative to the addition of Au-D-S-ssA (20 nM, $25 \mu \mathrm{L}$ ) to a dispersion containing Au-D-S-ssA' (20 nM, $25 \mu \mathrm{L}$ ) and 950 íL of Buffer B and Buffer C at $100 \mathrm{mM}$ salt concentration). (b) Average hydrodynamic diameter as a function of time for an aggregating dispersion of Au-D-S ssA and Au-D-SSSA' at various salt concentrations. (c) A log-log plot of rates of aggregation for various salt concentrations. (Note: The rates of aggregation correspond to the slopes of the graphs for the first $1000 \mathrm{~s}$ in Figure 3b.)

Addition of $\mathbf{S S A}^{\prime}$ to Au-D-S-ssA, at a salt concentration of $100 \mathrm{mM}$, resulted in an increase in size of approximately $4 \mathrm{~nm}$ (Figure 1d). This is a result of the binding of the biotinhexaethylene glycol-oligonucleotide, ssA', to the DNA immobilized on the gold nanocrystals, ssA. The increase in size by $4 \mathrm{~nm}$ can be attributed to the biotin-hexaethylene glycol moiety of the ssA'. Further addition of Au-D-S-ssA' had no significant effect on the size. This implies that the immobilized ssA has already formed duplexes with the ssA', hence Au-DS- $\mathbf{s s A}^{\prime}$ is unable to bind to Au-D-S-ssA and form duplexes (NOTE: This experiment is graphically illustrated in Supporting Information). A similar experiment, whereby Au-D-S-ssA was also added

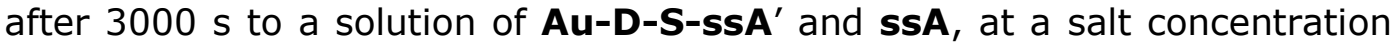
of $100 \mathrm{mM}$, yielded similar results. 
Several nonspecific interactions involving streptavidin could lead to an increase in the observed size due to the "sticky" nature of the protein.41 To account for this possibility, an equimolar concentration of Au-D-S-ssA was added to a dispersion of streptavidin-coated gold, Au-D-S, at $100 \mathrm{mM}$ salt concentration. The size increased from 28 to $36 \mathrm{~nm}$ (Figure 1d) and remained constant over time. This value is an average of the average hydrodynamic diameter for both Au-D-S-ssA (42 nm) and Au- D-S (28 $\mathrm{nm}$ ). Thus, no significant increase in size is observed as a result of the addition of DNA-modified gold to the "sticky" streptavidin-coated gold. Similar results were obtained for the addition of Au-D-S-ssA' to Au-D-S under similar conditions.

Hence, it can be concluded that any streptavidin unbound by ssDNA oligomers will not affect the aggregation process.

Controlling the Extent of Nanocrystal Aggregation. Having established that duplex formation is driving the aggregation process, it was expected that the aggregation of a dispersion of Au-D-S-ssA and Au-D-SSsA', at a salt concentration of $100 \mathrm{mM}$, would be rapidly inhibited by the addition of an excess of either ssA or $\mathbf{S S A}^{\prime}$ (Figure 2a). This is a consequence of the ssDNA oligomers possessing higher diffusion coefficients and diffusing at a faster rate to the DNA immobilized on the gold nanocrystals compared to the DNA-modified nanocrystals, which possess lower diffusion coefficients.

It is also clear that the average aggregate size depends on the time elapsed between the initiation of aggregation and the time that a monolayer equivalent of ssDNA oligomers is added to the aggregating colloid. Hence, it should be possible to determine the average aggregate size, depending on the time of addition of ssDNA.

Shown in Figure $2 a$ is the addition of SSA oligomers to an aggregating colloid of Au-D-S-ssA and Au-D-S-ssA'. It is found that nanocrystal aggregation can be inhibited almost immediately at any stage of the aggregation process. Representative TEM images (Figures 2b-I, 2b-II, 2bIII) show that correspondingly narrower particle size distributions are also obtained, depending on the time of addition of the ssA oligomers. The size distributions become narrower due to the smaller aggregates present in the aggregating dispersion diffusing together to form larger aggregates, thereby narrowing the size distribution of the aggregates. A similar effect is also observed for the addition of $\mathbf{S S A}^{\prime}$ oligomers to an aggregating dispersion of Au-D-S-ssA and Au-D-S-ssA'.

However, it must be emphasized that as a result of the subjective nature of TEM, plus effects such as the evaporation rate of the solvent and interactions between nanoparticles and the substrate, measurement of the exact sizes of the aggregates may prove unreliable by TEM. Hence, the images shown are only representative and the principle insight gained from this TEM study is that relatively narrower size distributions are obtained as aggregation is terminated at later stages of growth. The TEM images, therefore, substantiate and provide qualitative information about the quantitative results obtained from DLS.

Controlling the Rate of Nanocrystal Aggregation. As can be seen in Figures $3 a$ and $3 b$, the rate of aggregation of Au- D-S-ssA and Au-D-S- 
ssA', can be controlled by varying either the nanocrystal or the salt concentration independently of one another.

Since the rate of DNA duplex formation is dependent on several variables, it would be expected that controlling one or more of these parameters would provide a means of controlling the kinetics of assembly.

Figure $3 a$ simply shows that the rate of aggregation, at a constant salt concentration of $100 \mathrm{mM}$, increases as the particle concentration is increased. A power-law dependence of the aggregate size on time was observed for the three different concentrations.

Figure $3 \mathrm{~b}$ corresponds to samples, at a constant particle concentration, aggregated at varying salt concentrations. The slopes of the graphs of these individual aggregation processes, which correspond to the rates of aggregation, were also plotted against the salt concentration for the first $1000 \mathrm{~s}$ of aggregation in a log-log plot (Figure 3c). Data obtained at later stages in the aggregation process were not taken into account as a result of inaccuracies in the calculation of the average hydrodynamic diameter for increasing aggregate sizes (42). The resulting plot reveals a linear increase in the rate of growth as the salt concentration is increased. The rate of aggregation, however, remained constant beyond a salt concentration of $100 \mathrm{mM}$. Hence, it can be seen that up to $100 \mathrm{mM}$ salt concentration, the rate of growth, which we can assume to be proportional to the rate of duplex formation between the DNA-modified nanocrystals, is dependent on the salt concentration. Above this particular concentration (i.e., at higher salt concentrations) the rate of growth remains constant. Therefore, the aggregation process is dependent on the salt concentration to a significantly lesser extent. Consequently, we can also assume that under these conditions the rate is much less dependent on the rate of duplex formation. These findings would suggest that two distinct kinetic regimes for growth exist.

Reaction versus Diffusion-Limited Aggregation Kinetics. Previous studies on colloidal aggregation have revealed a transition from Reaction Limited-Aggregation (RLA) to Diffusion- Limited Aggregation (DLA) for the irreversible growth of colloids (42). In such studies, the stability of charge-stabilized colloids is affected by the addition of salt, which reduces the extent of interparticle screening. The amount of salt added determines the kinetics of the subsequent aggregation process. At low salt concentration the aggregation is limited by the sticking probability upon approach of two nanocrystals and is slow, i.e., Reaction-Limited Aggregation (RLA). At high salt concentration, aggregation is limited solely by the Stokes diffusion of the aggregates and is fast, i.e., Diffusion-Limited Aggregation (DLA).

A crossover from RLA kinetics to DLA kinetics can be observed for DNA-mediated nanocrystal assembly as presented here. It can be seen in Figure $3 \mathrm{~b}$ that salt concentrations up to $100 \mathrm{mM}$ increase the rate of growth of nanocrystals. Hence, it is assumed that the rate of growth of DNAmodified nanocrystals, which is directly proportional to the rate of duplex formation, increases as the salt concentration increases, i.e., RLA kinetics. However, at salt concentrations greater than $100 \mathrm{mM}$, the rate of growth 
remains constant. Hence, it is assumed that the rate of duplex formation is no longer the limiting factor governing the kinetics of aggregation. In this regime, DLA kinetics are observed as it is assumed that the rate of aggregation is now dependent on the diffusion of DNA-modified nanocrystals.

This crossover from RLA kinetics to DLA kinetics, depending on the salt concentration, could potentially impact upon the ordering of aggregates formed. For aggregates formed under RLA conditions, the collision time between particles is longer than for particles aggregated under DLA conditions. As a result, this may allow a degree of restructuring among the aggregating particles, which would result in a more ordered structure. Likewise, particles aggregated under DLA conditions would be expected to give rise to a less ordered structure because relatively short collision times would diminish the opportunities for the aggregates to restructure. Studies to examine the relationship between the salt concentration and the resultant ordering of such assemblies using more conclusive techniques to probe the aggregate ordering are presently ongoing.

\section{Conclusions}

The preparation of biotinylated gold nanocrystals subsequently modified by the addition of streptavidin-DNA conjugates has been described.

It has been shown that the modified nanocrystals can be directly programmed to recognize one another and assemble in solution by combining separate sets of nanocrystals with complementary strands of DNA.

The extent of aggregation can be controlled by the addition of SSDNA oligomers to an aggregating colloid of the gold nanocrystals modified with complementary strands of DNA. The ssDNA oligomers rapidly form duplexes with the immobilized DNA and the aggregation process can be instantly terminated. Depending on the time of addition of the ssDNA oligomers, this enables one to limit the size of the aggregates formed.

The rate of formation of the resulting aggregates can be controlled by varying the salt and modified nanocrystal concentrations. Two distinct regimes of kinetic aggregation, Reaction- Limited Aggregation (RLA) and Diffusion-Limited Aggregation (DLA), are observed depending on the salt concentration.

Moreover, the approach adopted is modular, requiring only the relevant biotin linker chemistry to be developed for a given nanoparticle, and precludes unfavorable interactions between the DNA and the streptavidin-coated nanoparticle.

Acknowledgment. This research was supported by a grant from the Petroleum Research Fund (Grant No. PRF\# 32879-ACS). The Authors also thank Dr. Hakan Rensmo for helpful discussions and express their gratitude for the services provided by $D$. Cottell and the staff at the Electron Microscopy Centre, National University of Ireland, Dublin.

Supporting Information Available: Plot of the absorbance (260 nm) versus temperature dependence for equimolar dispersions of Au-D-S-ssA and $\mathbf{A u}-\mathbf{D}-\mathbf{S}-\mathbf{s S} \mathbf{A}^{\prime}$ aggregated at a range of salt concentrations to show that 
DNA is driving the assembly process. Also included is a schematic illustration of the experiment carried out in Figure 1d (Au-D-S-ssA:ssA'+ Au-D-S-ssA'). This information is available free of charge via the Internet at http://pubs.acs.org.

\section{References and Notes}

(1) Collier, C. P.; Vossmeyer, T.; Heath, J. R. Annu. ReV. Phys. Chem. 1998, 49, 371.

(2) Murray, C. B.; Kagan, C. R.; Bawendi, M. G. Annu. ReV. Mater. Sci. 2000, 545.

(3) Connolly, S.; Rao, S. N.; Rizza, R.; Zaccheroni, N.; Fitzmaurice, D. Coordin. Chem. ReV. 1999, 186, 277.

(4) Vossmeyer, T.; DeIonno, E.; Heath, J. R. Angew. Chem., Int. Ed. Engl. 1997, 36, 1080.

(5) Storhoff, J. J.; Elghanian, R.; Mucic, R. C.; Mirkin, C. A.; Letsinger, R. L. J. Am. Chem. Soc. 1998, 120, 1959.

(6) Taton, T. A.; Mirkin, C. A.; Letsinger, R. L. Science 2000, 289, 1757.

(7) Ryan, D.; Rao, S. N.; Rensmo, H.; Fitzmaurice, D.; Preece, J. A.; Wenger, S.; Stoddart, J. F.; Zaccheroni, N. J. Am. Chem. Soc. 2000, 122, 6252.

(8) Shenton, W.; Pum, D.; Sleytr, U. B.; Mann, S. Nature 1997, 389, 585.

(9) Korgel, B. A.; Fitzmaurice, D. AdV. Mater. 1998, 10, 661.

(10) Josephson, L.; Manuel Perez, J.; Weissleder, R. Angew. Chem., Int. Ed. 2001, 40, 3204.

(11) Taton, T. A.; Mucic, R. C.; Mirkin, C. A.; Letsinger, R. L. J. Am. Chem. Soc. 2000, 122, 6305.

(12) Xia, Y. N.; Rogers, J. A.; Paul, K. E.; Whitesides, G. M. Chem. ReV. 1999, 99, 1823.

(13) Taton, T. A.; Lu, G.; Mirkin, C. A. J. Am. Chem. Soc. 2001, 123, 5164.

(14) Demers, L. M.; Mirkin, C. A. Angew. Chem., Int. Ed. 2001, 40, 3071.

(15) Mann, S.; Shenton, W.; Li, M.; Connolly, S.; Fitzmaurice, D. AdV. Mater. 2000, 12, 147.

(16) Elghanian, R.; Storhoff, J. J.; Mucic, R. C.; Letsinger, R. L.; Mirkin, C. A. Science 1997, 277, 1078-1080.

(17) Willner, I.; Patolsky, F.; Wasserman, J. Angew. Chem., Int. Ed. 2001, $40,1861$.

(18) Grabar, K. C.; Smith, P. C.; Musick, M. D.; Davis, J. A.; Walter, D. G.; Jackson, M. A.; Guthrie, A. P.; Natan, M. J. J. Am. Chem. Soc. 1996, 118, 1148.

(19) Boal, A. K.; Ilhan, F.; DeRouchey, J. E.; Thurn-Albrecht, T.; Russell, T. P.; Rotello, V. M. Nature 2000, 404, 746.

(20) Cusack, L.; Rizza, R.; Gorelov, A.; Fitzmaurice, D. Angew. Chem., Int. Ed. Engl. 1997, 36, 848.

(21) Whaley, S. R.; English, D. S.; Hu, E. L. Nature 2000, 405, 665.

(22) Andres, R. P.; Bielefeld, J. D.; Henderson, J. I.; Janes, D. B.; Kolagunta, V. R.; Kubiak, C. P.; Mahoney, W. J.; Osifchin, R. G. Science 1996, 273, 1690.

(23) Mirkin, C. A.; Letsinger, R. L.; Mucic, R. C.; Storhoff, J. J. Nature 1996, 382, 607.

(24) Alivisatos, A. P.; Johnsson, K. P.; Peng, X. G.; Wilson, T. E.; Loweth, C. J.; Bruchez, M. P.; Schultz, P. G. Nature 1996, 382, 609. 
(25) Loweth, C. J.; Caldwell, W. B.; Peng, X. G.; Alivisatos, A. P.; Schultz, P. G. Angew. Chem., Int. Ed. 1999, 38, 1808.

(26) Mucic, R. C.; Storhoff, J. J.; Mirkin, C. A.; Letsinger, R. L. J. Am. Chem. Soc. 1998, 120, 12674.

(27) Mitchell, G. P.; Mirkin, C. A.; Letsinger, R. L. J. Am. Chem. Soc. 1999, $121,8122$.

(28) Watson, K. J.; Park, S.-J.; Im, J.-H.; Nguyen, S. T.; Mirkin, C. A. J. Am. Chem. Soc. 2001, 123, 5592.

(29) Niemeyer, C. M.; Burger, W.; Peplies, J. Angew. Chem., Int. Ed. 1998, $37,2265$.

(30) Niemeyer, C. M.; Ceyhan, B. Angew. Chem., Int. Ed. 2001, 40, 3685.

(31) Park, S.-J.; Lazarides, A. A.; Mirkin, C. A. Angew. Chem., Int. Ed. 2001, 40, 2909.

(32) Connolly, S.; Fitzmaurice, D. AdV. Mater. 1999, 11, 1202.

(33) Connolly, S.; Cobbe, S.; Fitzmaurice, D. J. Phys. Chem. B 2001 105, 2222.

(34) Smoluchowski, M. Z. Phys. Chem. 1917, 92, 129.

(35) Kisak, E. T.; Kennedy, M. T.; Trommeshauser, D.; Zasadzinski, J. A. Langmuir 2000, 16, 2825.

(36) Connolly, S.; Rao, S. N.; Fitzmaurice, D. J. Phys. Chem. B 2000, 104, 4765.

(37) Green, N. M. In Methods in Enzymology, 18A; McCormick, D. B., Wright, L. D., Eds.; Academic Press: New York, 1974; p 418.

(38) Cantor, C. R.; Warshaw, M. M.; Shapiro, H. Biopolymers 1970, 9, 1059.

(39) Weber, P. C.; Ohlendorf, D. H.; Wendoloski, J. J.; Salemme, F. R. Science 1989, 243, 85.

(40) Zubay, G. Biochemistry; Wm. C. Brown Publishers: Oxford, 1993.

(41) Bayer, E.; Wilchek, M. Methods Enzymol. 1990, 184, 201.

(42) (a) Weitz, D.; Olivera, M. Phys. Rev. Lett. 1984, 52, 1433. (b) Weitz,

D.; Huang, J.; Lin, M. Sung, J. Phys. Rev.. Lett. 1984, 53, 1657. (c) Weitz,

D.; Huang, J.; Lin, M.; Sung, J. Phys. Rev. Lett. 1985, 54, 1416. (d) Lin, M. Y.; Lindsay, H. M.; Weitz, D. A.; Ball, R. C.; Klein, R.; Meakin, P. Nature 1989, 339, 360. (e) Lin, M. Y.; Lindsay, H. M.; Weitz, D. A.; Ball, R. C.; Klein, R.; Meakin, P. Phys. Rev. A 1990, 41, 2005. (f) Lin, M. Y.; Lindsay, H. M.; Weitz, D. A.; Klein, R.; Ball, R. C.; Meakin, P. J. Phys. Condens. Mater. 1990, 2, 3093. (g) Zhou, Z.; Chu, B. J. Colloid Sci. 1991, 143, 356. (h) Di Biasio, A.; Bolle, G.; Cametti, C.; Codastefano, P.; Sciortino, F.; Tartaglia, P. Phys. Rev. E 1994, 50, 1649. (i) Di Biasio, A.; Bordi, F.; Cametti, C.; Codastefano, P. J. Chem. Soc., Faraday Trans. 1998, 94, 3477. (j) Odriozola, G.; Tirado-Miranda, M.; Schmitt, A.; Lopez, F. M.; Callejas-Fernandez, J.; Martinez Garcia, R.; Hidalgo-Alvarez, R. J. Colloid Sci. 2001, 240, 90. 


\section{SUPPORTING INFORMATION}

\section{Effect on Tm by varying $[\mathrm{NaCl}]$}

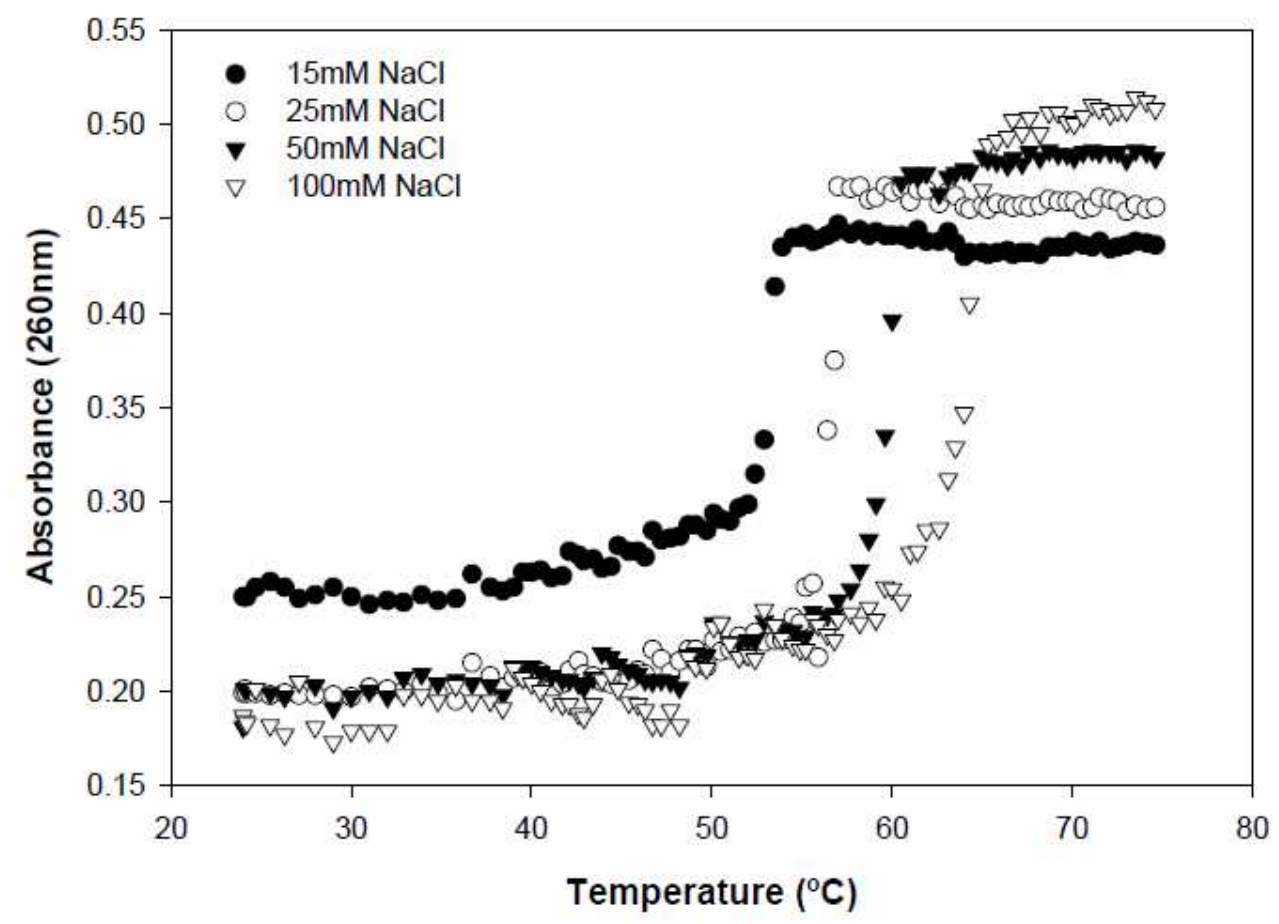

A UV study of the effect on the DNA transition melt temperatures of equimolar dispersions of Au-D-S-ssA and Au-D-S-ssA', aggregated at a range of salt concentrations, $t$ o show that DNA is driving the assembly process 
Illustration of experiment carried out in Figure 1(d)
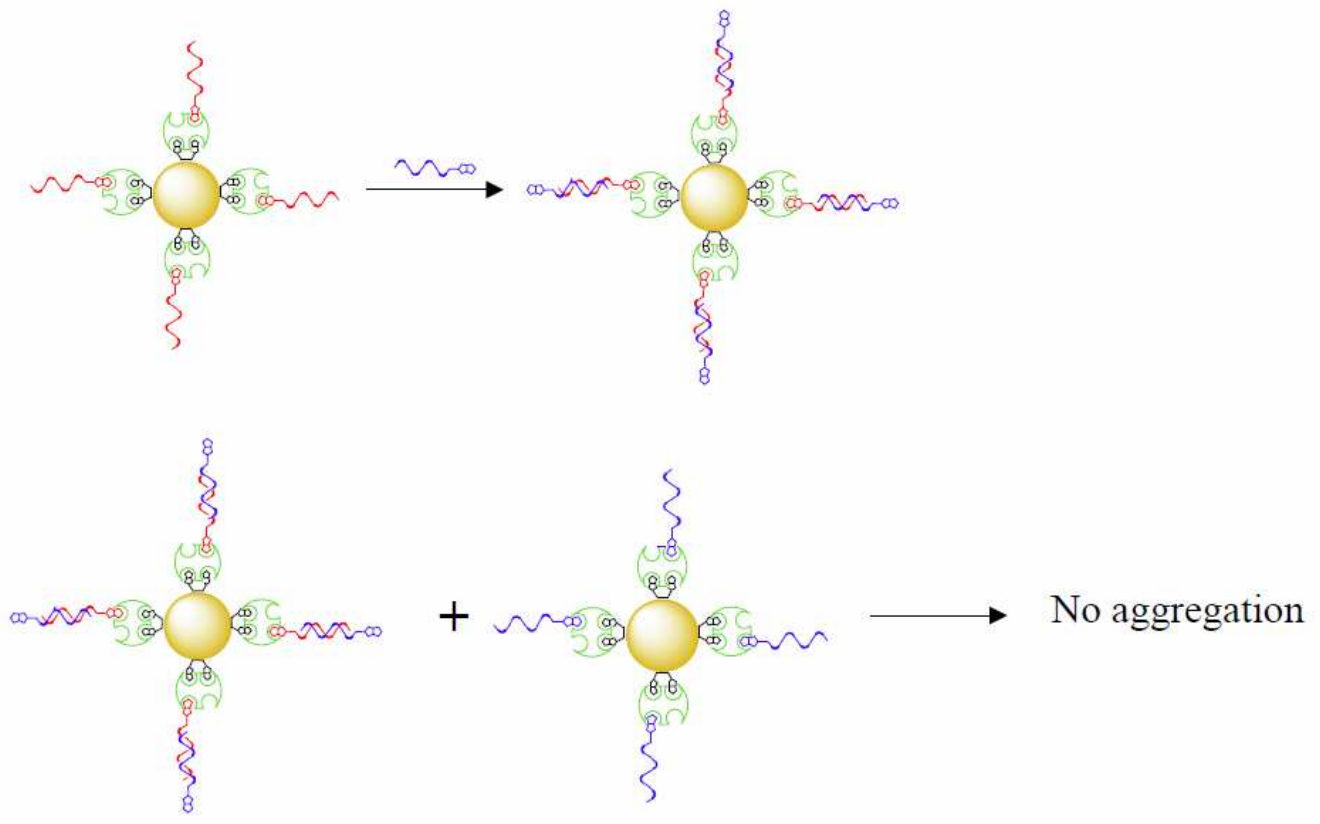